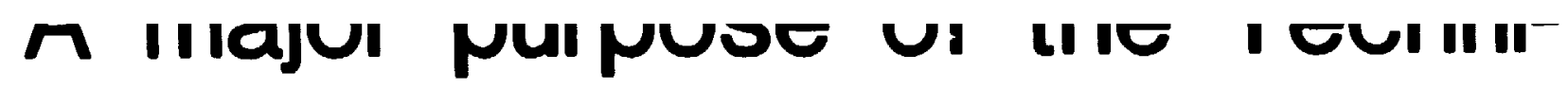
cal Information Center is to provide the broadest dissemination possible of information contained in DOE's Research and Development Reports to business, industry, the academic community, and federal, state and local governments.

Although a small portion of this report is not reproducible, it is being made available to expedite the availability of information on the research discussed herein.
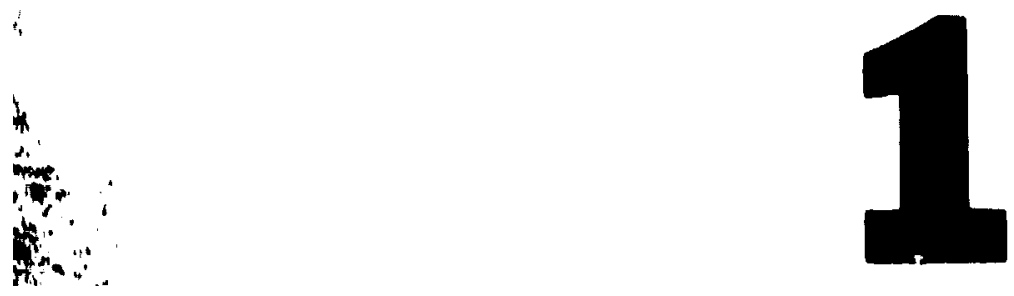
LA-UR- $-85-4265$

DE86 004733

TITLE AN APPROACH TO ERROR-ESTIMATION IN THE APPLICATION OF DIMENSION ALGORI THMS

AUTHOR(S). Joachim Holzfuss and Gottfried Mayer-Kress

SUBMITTED TO. CNLS Workshop "Dimensions and Entropies in Chaotic Systems" Proceedings held Sept. 11-16, 1985 at the Pecos River Conference Center

\section{DISCLAIMER}

This report was proparat as an acoount of work aponeored by an agency of the United Statea Goversment. Neither the United Siates Gnvernment nor any asency thereof, nor any of their employeos, makes any watrenty, expreas ur Implied, or asumen any logal liabillty or responalbility for the securacy, complotones, or usefulness of any information, apparatus, product, of process disclowed, of represents that its use would nol infringe privately owned sighte. Rofor. ence herein to any spocific commercial product, procen, or corvice by trade namo, trademark, manufacturer, or otherwise does not necousarily conatitute of imply its endorseinent, recommendation, of favoring by the United States Government of any agency thereof. The viewa and opinione of authure expreased herein do not neccessarily state or ruflect thase of the United Statea Covernment of any agency thereof. 


\title{
An Approach to Error-Estimation in the Application of Dimension Algorithms
}

\author{
joachim Holzfuss" and Gottfried Mayer-Kress \\ Center for Nonlinear Studies, MS B258, Los Alamos National Laboratory \\ Los Alamos, N.M. 87545, USA
}

\author{
- Permanent address: Drittes Physikalisches Institut, Universitaet \\ Goettingen, D-3400 Goettingen, Fed. Rep. of Germany
}

Three different methods for calculating the dimension of attractors are analyzed. An approach to error-estimation is presented and is used on various datase's. It is show'n, that in some cases the errors can become very large.

\section{Linlroduction}

The dimension of atcractors reconstructed from a tirse series [1] is of sreat physical interest especially in experimental situations $[2,3]$. It is a measure for the number of active modes modulisting a physical process and therefore a measure of complexity. Many different methods $[4,5,6)$ of calculating the dimension of attractors have been introduced. Very important questions are, bow far these methods are reliable and how large the uncertainty of a calculated dimension is. Most of the algorithms used for dimension meusurements of attractors, reconstructed from numerical and experimental data, average over certain variables, such as the number of nearest neighbors, the mass of a cube of a certain sidelength or pointwise dimensions from different refurence points on the attractor. All these averages must be taken into account, if one wishes 10 determine $a$ realistic error estimate of a fractal dimension. In the literature error estimates are mainly calculated by just everaging over some values of the scaling exponents obtained in different length scales (least squares fit). This method can truly underestimate existing errors. The errors can make it useless for experimentalists to deal with smaller/larger relationships between different definitions of fractal dimensions. The largest error source is the limitation in the number of data avalable to reconstruct an attra:tor from a time series. The number of data points necensary for filling this subset of a phase apace with points to get the anme probability messure a given by the attractor of the physical process might be very large and even increases exponentially with the dimension of the attractor. This also gives rise to the question, whether an andysis of high dimensional attractors is possible.

We fint recall some different methode for celculating the dimenoion (II), and then introduce cur approach to delermine the error (III). Niter a short description of the computer prozrams and come ideas for automation of the 
dimension calculation ( $N$ ), we analyze diferent data sets, including a 5-torus, gaussian noise and the Lorenz attractor (V).

\section{Methods for Celculatins the Dimension of an Autractor}

To reconstruct an attractor from a time series of a single probe, we use the now classical method of time delay coordinates. In this method a vector $\boldsymbol{X}\left(t_{k}\right)$ in an $n$-dimensional phase space is constructed by taking delayed samples of the time series $I\left(t_{k}\right)$ as coordinates [1], such that

$$
\boldsymbol{X}\left(t_{k}\right)=\left(x\left(t_{k}\right), x\left(t_{k}+T\right), x\left(t_{k}+2 T\right), \cdots, x\left(t_{k}+(n-1) T\right)\right)
$$

where $t_{k}$ is the discrete time with $k$ running from 1 to the number of data points and $T$ is an 'arbitrary', but fixed time delay. The embedding dimension $n$ is the number of coordinates of the embedding space. If $T$ is chosen to be equal to the time delay, where there is a minimum in the mutual information between two measurements (7), a D-dimensional attractor is construct.ed best by taking the embedding dimension larger than $2 D+1$. For aigals with strong periodic contents this time delay is approximately equal to the first zero-croasing of the autocorrelation function.

We used three different methods for calculating the "fractal" dimension of an attractor.

1. The pointwise dimension (mass dimension) $[4,8]$ can be defined as

$$
D_{r}=\lim _{n_{\text {deto }} \rightarrow \infty} \lim _{r \rightarrow 0} \frac{\log \frac{1}{n_{\text {dase }}} N_{x_{0}}(r)}{\log r}
$$

and it consists of counting the number of data points $N_{X_{0}}(r)$ witbin a cube of sidelength $r$ centered at a point $\vec{X}_{0}$ on the attractor. Due to the fact, that one does not have an infinite amount of data points and also no infinite precision, as required in the definition, one has 10 average over severd reference points $\vec{X}_{0}$. Fig. 1 shows the scaling behavior of $N_{X_{0}}(r)$ of different reference points. We calculate the pointwise dimensions for 200 reference points and take the average, which yields a good estimate for the dimension of the attractor.

2. Another method we used was the determination of the correlation dinension $D_{2}$ with the algorithm proposed by GRASSBERGER and PRO. CACCLA $[5 \mid$. They showed the scaling of the correlation integral $C(r)$ for small $r$

$$
C(r) \cdot r^{D}
$$

with 


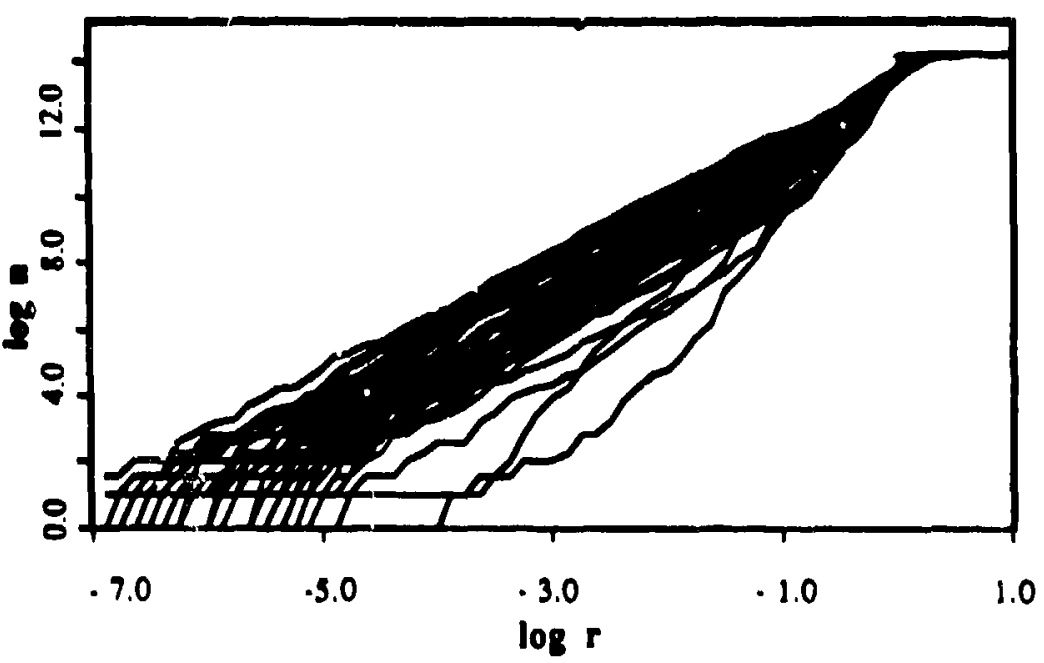

Fig 1: Scaling behavior of $N_{X_{0}}(r)$ at 60 reference points $X_{0}$ on the Lorenz attractor. The embedding dimension is 5 . 


$$
C(r)=\lim _{n_{\text {date }} \rightarrow \infty} \frac{1}{n_{\text {rel }}} \sum_{j=1}^{n_{r e f}} \frac{1}{n_{\text {date }}} \sum_{i=1}^{n_{\text {data }}} \theta\left(r-\left|X_{i}-X_{j}\right|\right)
$$

and $\theta$ equal to 1 for positive and 0 for negative arguments.

Also

$$
C(r)=\lim _{n_{\text {data }} \rightarrow \infty} \frac{1}{n_{r e s}} \sum_{j=1}^{n_{r e f}} \frac{1}{n_{\text {tade }}} N_{X_{j}}(r)
$$

with $N_{X}$ equal to the rightmost sum in eq. (4). $C(r)$ counts the number of points $N_{X},(r)$ in a cube of fixed sidelength $r$, averages over all the cubes, that are centered at different reference points $\vec{X}_{j}$, and normalizes. An example shows, how the averaging is done.

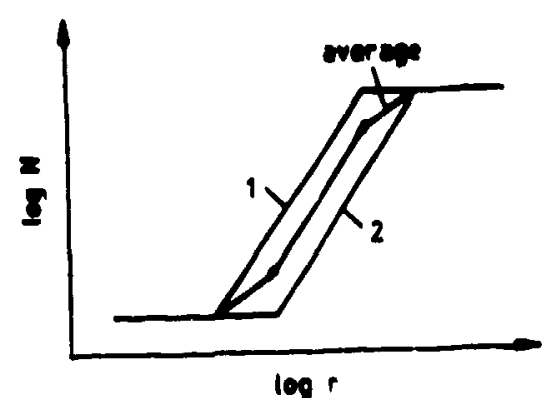

Fig. 2: sketch of the scaling behavior for 2 reference points with different scaling regions $\left[r_{\text {min }}, r_{\text {max }}\right.$ ] and their average (Grassberger and Procaccia method).

The scaling of the contents of the cubes with respect to $r$ in two different regions on the attractor (fig. 2) is discribed by

$$
N_{1}(r)=\left(\alpha_{1} r\right)^{D} \quad \text { and } \quad N_{2}(r)=\left(\alpha_{2} r\right)^{D} \quad \alpha_{1}, \alpha_{2}=\text { conot. }
$$

The Grassberger and Procaccia algorithm averages over the $N$-vaiues, while $r$ is fixed:

$$
\begin{array}{r}
C(r)=\frac{N_{1}(r)+N_{2}(r)}{2}-\frac{1}{2} \\
N(r)=r^{D}\left(\frac{a_{1}^{D}+\alpha_{2}^{D}}{2}\right)
\end{array}
$$

Fol infinitely expanded scaling behavior we get the same scaling exponent $D$ for the averaged values. Large differences in the $\alpha$ 's and amall scaling regions distort the scaling properties of $C(r)$.

3. The third method considered here, is the one oroposed by TERMONLA and ALEXANDROWICZ [B], It consists of averaging over the different radii of 
cubes, which contain a fixed number of data points. They showed that $N$, the 'number of nearest neighbors', behaves like

$$
N \cdot \bar{r}(N)^{D_{r}} \text {, }
$$

where $F(n)$ is the average radius of the cubes containing $N$ data points and $D_{F}$ ' the "fractal" dimension of the attractor. As an example we consider again the scaling behavior for two reference points, this time keeping $N$ fxed and $r$ variabel.

$$
N=\left(\alpha_{1} r_{1}\right)^{D} \quad \text { and } \quad N=\left(\alpha_{2} r_{2}\right)^{D} \quad \alpha_{1}, \alpha_{2}=\text { conot }
$$

Then

$$
\frac{N^{\frac{1}{D}}}{\alpha_{1}}=r_{1}(N) \text { and } \frac{N^{\frac{1}{D}}}{\alpha_{2}}=r_{2}(N)
$$

and

$$
N^{\frac{1}{D}}\left(\frac{1}{2}\left(\frac{1}{a_{1}}+\frac{1}{\alpha_{2}}\right)\right)=\frac{r_{1}(N)+r_{2}(N)}{2}=\bar{F}(N)
$$

gives

$$
N=\bar{r}(N)^{D}\left(\frac{1}{2}\left(\frac{1}{\alpha_{1}}+\frac{1}{\alpha_{2}}\right)\right)^{-D} \quad,
$$

which results in the same scaling exponent $D$ for the averaged values as for the single reference points.

All three methods use a different kind of averaging. The last two methods have one thing in common: they average over an ensemble of single values ( $N$ in case 2, $r$ in case 3 ) and don't care about the crientation of the lines in fig. 1 i.e. the scaling benavior of the attractor at ths different reference points. This may in some cases lead to a misinterpretation of the results, if the averaged values pretend a scaling behavior, that the values obtained from different reference points may have never had. In method 1 lise aditional information of the reference points is used when the aversged pointwise dimension is calculated.

\section{Error Estimation}

Each value of the pointwise dimension $D_{\text {a }}$ was obtained by calculatiog the slope of a fited straight line to each curve in the $\log r / \log N$ plot using least squares fit. In order 10 determine the spread of values of the $D_{*}$ we consider their standard deviation, given by

$$
\Delta D=\sqrt{D^{2}-D^{2}}
$$

which saj:, that $68.3 \%$ of all valijes of a normal distribution lie in the interval $(D-\Delta D, D+\Delta D)$. 
In case 2 and 3 straight lines are also fitted to the values in the doubly logarithmic plot using least squares fit. The least squares fit consists of finding a straight line

$$
y_{i j}=a+b x_{i j}+z_{i j}
$$

such that $\sum\left(z_{i j}\right)^{2}$, the sum ol squares of the errors $z_{i j}$ is minimized. The index $j$ denotes the different reference points and the index $i$ their average value. It is also possible to use weighted least squares fit, which minimizes

$$
\sum w_{i} z_{i}^{2}=\sum w_{i}\left(y_{i}-a-b x_{i}\right)^{2}
$$

with

$$
w_{i}=\frac{1}{\Delta^{2} y_{i}}
$$

and

$$
y_{i}=\frac{1}{n_{r e l}} \sum_{j=1}^{n_{r e f}} y_{i j}
$$

$z_{i}$ and $x_{i}$ have the same definition. Using wejahted least squares fit reduces the weight of values, if their variance $\Delta^{2} y_{i}$ is large. However, because of the finite size of the attractor, the variances become very small for large values of $\log r$ (saturation region) and therefore could give rise to a false $6 \mathrm{t}$. Therefore we have to exclude this possibility by e.g. imposing a lower bound for the slope, which shouid be fited or by restricting the possible acaling ranges.

In terms of the Grassberger and Procaccia algorithm $y_{i}$ is the average of the $\log N_{i j}$ values of a hxed radius $r_{i}$ over all reference points $\vec{X}_{j} \quad$ (fig. $3 \mathrm{a}$ ). In the Termonia and Alexandrowicz method $y_{i}$ wor'd be the average of the logarithms of the radii $\operatorname{lng}_{g} r_{i j}$ with a fixed number of nearest neighbors $N_{i}$ (fig. 3b) .

We use the average over the logarithmic values in determining $\Delta^{2} y_{i}$ and minimize the errors $s_{i j}$ of the straight line in the $\log r / \log N$ plos. This gives a good approximation of the fully consistent way of averaging over the nonlogarithmic values of $r$ or $N$, which would require a minimizing of

$$
\sum_{i}\left(y_{i j}-a x_{i j}^{b}\right)^{2}
$$

In order to set the standard deviation of the slope, we have $\Delta y+\Delta z=(b \Delta x)+(\Delta a+\Delta b \Delta x)$. We exclude possible parallel shiftings of the lines obtained at the different refrence points $u$ be able 10 evaluate the largest posaible error of the slope. because methods 2 and 3 yield no information about the orientation of these lines. This is done by setting $\Delta a$ to zero. Now we get for the standard deviation of the slope 


$$
\Delta b=\frac{\Delta z}{\Delta x}
$$

$\Delta z$ is the standard deviation of the differences of the actual $y-v a l u e s$ to the $y$-values of the fitted line. $\Delta x$ is the standard deviation of the $x$-values. From fg. $4 \mathrm{a}, \mathrm{b}$ we see, that $\Delta b$ is the standard deviation of all the possible values of the slope $b$.
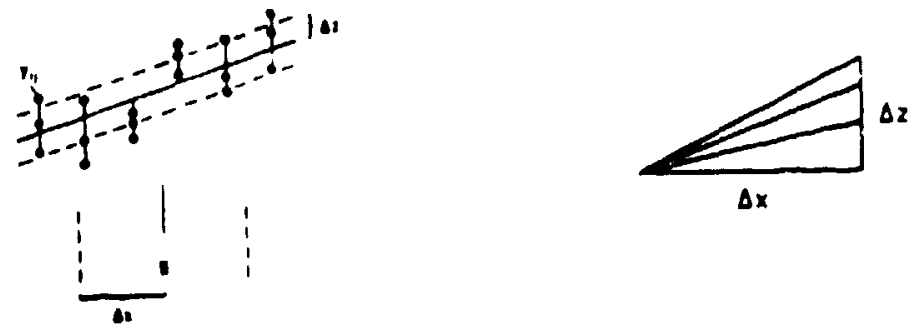

Fig. $4 a$ : standard deviation of $x-v a l u e s$ and of the errors 2 of the $y-v a l u e s$

$b$ : the possible changes of the slope $b$

In case 2 the errors are symmetric around the average slope. In case 3 , where we calculate $r(N)$ and therefore get an error for $\frac{1}{D}$, they get asymmetric when solving for the slope $D$.

To modify the definition into a better computable form we have

$$
\Delta^{2} b=\frac{\Delta^{2} z}{\Delta^{2} x}=\frac{\overline{z^{2}}-\bar{z}^{2}}{\overline{z^{2}}-\bar{x}^{2}}
$$

Due to the least squares fit $\bar{z} \approx 0$. Taking the average between the differences of all the $\log r$ or $\log N$ values and the values of the straight line, which is fitted to the logarithms of the averages of the nonlogarithmic $r$ or $N$-values implies a very small correction $\bar{z} \mid=0$, which can be neglected. Therefore we get

$$
\Delta^{2} b=\frac{\sum_{i=1}^{n_{\text {rad }}} \sum_{j=1}^{n_{r e f}}\left(y_{i j}-a-b x_{i j}\right)^{2}}{n_{r=1} \sum_{j=1}^{n_{r e f}}\left(x_{i j}-\bar{x}\right)^{2}}
$$

where $n_{\text {rel }}$ is the number of reference points and $n_{\text {rad }}$ is the number of the averaged values of distances $r$ sub $i$. With the average over all reference points (17), its variance is given by: 


$$
\Delta^{2} y_{i}=\sum_{j=1}^{n_{r e f}} \frac{\left(y_{i j}\right)^{2}}{n_{r e s}}-\left(\sum_{j=1}^{n_{r e f}} \frac{y_{i j}}{n_{r e f}}\right)^{2}
$$

and with

$$
\begin{gathered}
\sum_{i=1}^{n_{\text {red }}} \sum_{j=1}^{n_{\text {ref }}}\left(x_{i j}-\bar{x}\right)^{2}=n_{\text {res }} \sum_{i=1}^{n_{\text {rad }}}\left(x_{i}-\bar{x}\right)^{2} \\
\left(x_{i j}=x_{i} \text { f.a. } j\right)
\end{gathered}
$$

we get

$$
\Delta^{2} b=\frac{\sum_{i=1}^{n_{r a d}} \Delta^{2} y_{i}+\sum_{i=1}^{n_{r a d}}\left(y_{i}-a-b x_{i}\right)^{2}}{\sum_{i=1}^{n_{r a d}}\left(x_{i}-\bar{x}\right)^{2}}
$$

This expression allows to compute the variances of all the $y_{i}$ 's first and to get the variance of the slope when fitting over a certain number of $y_{i}$ 's. Expression ( 22 ) can also be modified by using

$$
a=\bar{y}-b \bar{x}
$$

and

$$
b=\frac{\sum_{i=1}^{n_{r a d}} \sum_{j=1}^{n_{r e f}} \frac{x_{i j} y_{i j}}{n_{r e d} \cdot n_{r e l}}-\bar{x} \bar{y}}{\Delta^{2} x}
$$

with $\bar{x}$ and $\bar{y}$ equal to the grand mean into

$$
\Delta^{2} b=\frac{\Delta^{2} y}{\Delta^{2} x}-b^{2}
$$

The variances are taken over all $n_{\text {red }} * n_{\text {ref }}$ points.

\section{Discriotion of the Prosrams}

The programs we use are designed for a fully automatic analysis of the data sets. First of all the program has to find the scying region. This is done by looking for the interval of a given length in the $\log r / \log N$ plot, where the root-mean-square error of the deviation from a fitted straight line, which is defined as (see also eqn. (16))

$$
\sqrt{\frac{1}{n_{r e d}} \sum_{i=1}^{n} w_{i} z_{i}^{2}}
$$


is the smallest. In case 1 , where we consider the averaged pointwise dimension, the weights $w_{i}$ are all 1 . Here the program calculates the slope of a fitted line at each reference point. Then it averages over just $20 \%$ of the obtained values of the pointwise dimensions, neglecting all the curves, that don't show a scaling behavior over the entire range of $r$-values. After calculating the average and the standard deviation it repeats these steps in a different embedding dimension. In case 2 and 3 the procedure is about the same, except there is just one line to be fitted. Also the weights are set to their respective values (eqn. 17). The procedure also features a "self-blowup" of the length of the fitted line: If the wotal length of the scaling region is unknown, the program starts with a given short interval length and finds the scaling region by minimizing the RMS error (eqn. 29). Then it repeats this step with an enlarged interval until a certain threshold value of the KMS error is reached, thus indicating, that the fitted interval length exceeds the length of the scaling region. In ail cases we find that 0.05 is a "good" threshold value.

\section{Analysis of Different. Datasets}

In the analysis the total number of data points is always 20,000 . We average over 200 reference points. The time delay $T$ for the reconstruction of the attractor is chosen according to reference 7 by calculating the mutual information. As the first example we consider a 5-torus, constructed from a time series with a Fourier spectrum of 5 incommensurate frequencies. We analyze the data with all three methods (fig. $5 a, b, c)$. In all three figures we see, that the calculated dimension of the attractor converges with increasing embedding dimension, and it has the value $D=5$ from sbout $2 D+1=11$. The standard deviations of the attractor dimension, described by the error bars, converge also to certain values. For the averaged pointwise dimension we get $5+/-0.3$, for the correlation dimension $5+/-0.5$ and for the dimension obtained by the TERMONIA/ALEXANDROWICZ [6] method $5+0.8 /-0.7$.

The calrulation of the dimension of the Lorenz attractor (fig $6 \mathrm{a}, \mathrm{b}, \mathrm{c}$ ) show's also good convergence at aboui $2 D+1$. The val ies for $D$ cbtained by the different methods were $2+/-0.15$ in the case of the averaged pointwise dimension, $2+/-0.6$ in case 2 and $2+0.6 /-0.4$ in case 3 . The growing of the standard deviation in case 2 and 3 is due to successive shortening of the scaling region in higher embedding dimensions becsuse of geometrical effects. Smaller scaling regions with constant variance at each average value in the $\log r / \log N$ diagram result in a larger variance of the slope of the fitted line. The average of the pointwise dimension is not affected by this, when the length of the fitted line equals the length of the scaling region.

In the third example we analyzed gaussian noise, to have an easy example of higher dimensional attractors $(\mathrm{f} g, 7 \mathrm{a}, \mathrm{b}, \mathrm{c})$. Noise is considered to be spacefilling, i.e. each phase space of every embedding dimension is filled. Each one of the three methods couldn't produce this result. This is truly seen in the 
deviation from the $15^{\circ}$ line. The deviation is due to the increasing amount of data necessary for calculatiag bigher dimensions. Also it ean be extracted, that the error inereases almost linearly with the attractor dimension. Compared to the averaged pointwise dimension, the dimension calculated with the two other methods showed a very large possible error.

\section{Conclusions}

Three different methods of exieulating the dimension of attractore were analyzed. To each of those an approach to estimate the error was presented, which was based on calculating otendard deviations for certain variablef. Examples of analyzed data sets showed, that the averaged pointwise dimension provided the smallest possible error in the calculated dimension. It alsc seemed to be closer to the real value. It was shown, that the possible errors in the methods of GRASSBERGER/ PROCACCLA [5] and TERMONIA/ ALEXANDROWICZ [6] can be very large. High dimensional analysis is shown to be very dificult, because of the linear growth of the errors with the embedding dimension.

\section{Acknowledrements}

J.H. wants to thank the Center for Nonlinear Studies for the hospitality and financial support and $W$. Lauterborn for very useful discussions. We are deeply indepted to Erica Jen for permission of using versions of her codes which are the basis of parts of our numerical calculations. We also appreciate very helpful discussjons with Erisa Jen and J. Doyne Farmer. All computations were done in Los Alamos on CRAY 1 computers.

References:

1 Packard, N. H.,Crutchfield, J. P., Farmer, J. D., Shaw, R. S.: Geometry from a Time Series. Phys. Rev, Lett. 45, $\theta$ (1880) 712

2 Brandataetter, A., Swift, J.,Swinney, H. L., Wolf, A., Farmer, J. D., Jen, E., Crulchfield, P. J.: Low-Dimensional Chsos in a Hydrodynamic System. Phys. Rev. Lell. 51,16 (1983) 1442 
3 Lauterborn, W., Holzfuss, J.: Evidence for a Low-Dimensional Strange Attractor in Acoustic Turbulence. Preprint Gottingen

1 Farmer, J. D., Ott, E. and Yorke, J. A.: The Dimension of Chaotic Attractors. Physici 7D (1983) North Holland 153-180

5 Grassberger, P. and Procaccia, I.: Characterization of Strange Attractors. Phys. Rev. Lett. 50, 5 (1983) 346

6 Termonia. $\because$ and Alexandrowicz, Z.: Fractal Dimension of Strange Attractors. Pbys. Rsv. Lett. 51, 14 (1983) 1265

7 A. Frazer, this volume

8 Manderbrot, B.: The Fractal Geometry of Nature (W. H. Freeman and Co., San Francisco, 1882). 